\title{
Future Hypernuclear Program at JLab Hall C
}

S.N. Nakamura ${ }^{a}$, O. Hashimoto, Y. Fujii, H. Tamura, T. Takahashi, K. Maeda, H. Kanda, Y. Okayasu, H. Nomura, A. Matsumura, M. Oyamada, D. Honda, A. Ohtani, F. Kato, T. Watanabe, K. Tsukada, M. Ukai, Y. Miura, H. Yamauchi, S. Kato, Y. Sato, H. Noumi, T. Motoba, L. Tang, O.K. Baker, M. Christy, L. Cole, P. Gueye, C. Keppel, A. Uzzle, L. Yuan, J. Reinhold, P. Markowitz, B. Beckford, M. Carl, S. Gullon, C. Vega, Ed.V. Hungerford, T. Miyoshi, K. Lan, N. Elhayari, N. Klantrains, Y. Li, M. Buhkari, S. Radeniya, R. Carlini, R. Ent, H. Fenker, D. Mack, G. Smith, W. Vulcan, S. Wood, C. Yan, N. Simicevic, S. Wells, L. Gan, A. Ahmidouch, S. Danagoulian, A. Gasparian, D. Dehnhard, M. Elaasar, R. Asaturyan, H. Mkrtchyan, A. Margaryan, S. Stepanyan, V. Tadevosyan, D. Androic, T. Petkovic, M. Planinic, M. Furic, T. Angelescu, V.P. Likhachev, M. Ahmed

\section{JLab E01-011 Collaboration}

${ }^{a}$ Department of Physics, Tohoku University, Aoba, Sendai, Miyagi, 980-8578, Japan

Encouraged by the success of the first hypernuclear spectroscopy through the $\left(\mathrm{e}, \mathrm{e}^{\prime} \mathrm{K}^{+}\right)$ reaction (JLab E89-009), a new improved experiment with a newly developed High resolution Kaon Spectrometer (HKS) and new configuration of the electron spectrometer is planned at the JLab Hall-C. The introduction of the HKS will improve the energy resolution which was limited by the kaon spectrometer by a factor of two. The hypernuclear yield and the signal to noise ratio will be also improved by a factor of 50 and 10 , respectively.

\section{Introduction}

So far, $\Lambda$ hypernuclei have been extensively studied by using the meson-induced reactions such as $\left(\pi^{+}, \mathrm{K}^{+}\right)$and $\left(\mathrm{K}^{-}, \pi^{-}\right)$. The $\left(\mathrm{e}, \mathrm{e}^{\prime} \mathrm{K}^{+}\right)$reaction is new as a method for the hypernuclei spectroscopy, and it has unique advantages over those through meson-induced reactions. For example, the $\left(\mathrm{e}, \mathrm{e}^{\prime} \mathrm{K}^{+}\right)$reaction favorably excites spin-flip $\Lambda$ hypernuclear states and produces neutron rich $\Lambda$ hypernuclei converting a proton to a $\Lambda$ hyperon. From the experimental point of view, it is a great advantage that a high-quality electron beam allows us to improve the energy resolution down to sub-MeV.

The first $\left(\mathrm{e}, \mathrm{e}^{\prime} \mathrm{K}^{+}\right)$hypernuclear spectroscopy experiment was carried out successfully at the JLab Hall C in the spring of 2000 by the E89-009 collaboration. This pioneer experiment demonstrated a great potential of the $\left(\mathrm{e}, \mathrm{e}^{\prime} \mathrm{K}^{+}\right)$reaction, obtaining a hypernuclear mass spectrum with an energy resolution of about $900 \mathrm{keV}(\mathrm{FWHM})$ in the ${ }^{12} \mathrm{C}\left(\mathrm{e}, \mathrm{e}^{\prime} \mathrm{K}^{+}\right)_{\Lambda}^{12} \mathrm{~B}$ reaction[1]. However, the obtained data suffer from poor signal to noise ratio $(\sim 1)$ and limited statistics. It is clear that an improvement of this hypernuclear spectrometer sys- 
tem is essential to fully explore the potential of the new reaction to study hypernuclear structure.

For this purpose, the E01-011 collaboration proposed a new experiment with a newly developed HKS spectrometer which is designed for the $\left(\mathrm{e}, \mathrm{e}^{\prime} \mathrm{K}^{+}\right)$hypernuclear spectroscopy.

\section{Experimental principle}

Figure 1 shows a schematic view of the E01-011 setup. The basic configuration of the E01-011 experiment is similar to that of E89-009. The beam energy $(1.8 \mathrm{GeV})$ was chosen as low as possible not to open unnecessary reaction channels and to make the virtual photon energy at $\sim 1.5 \mathrm{GeV}$; the $\mathrm{p}\left(\gamma, \mathrm{K}^{+}\right) \Lambda$ cross section decreases for $E_{\gamma}>1.5$ $\mathrm{GeV}[2]$.

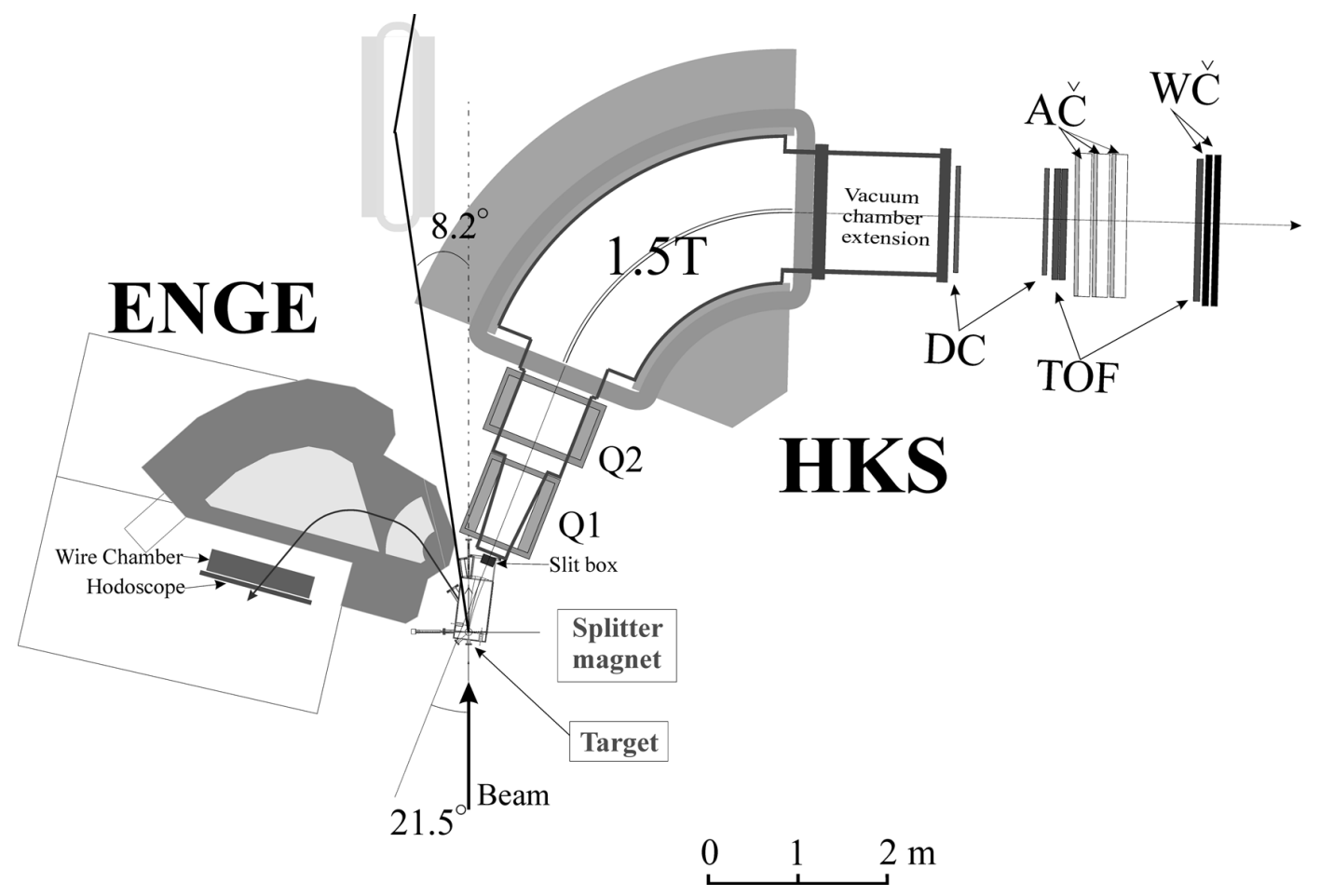

Figure 1. Schematic figure of the E01-011 setup.

The scattered electron (e') and $\mathrm{K}^{+}$are separated by the dipole magnet (Splitter) located just behind the target. The scattered electron is measured by the Enge split-pole spectrometer (ENGE) which was also used for the E89-009 experiment and $\mathrm{K}^{+}$by a newly developed high resolution kaon spectrometer (HKS).

The two key experimental conditions were improved from the previous experiment: 1) introduction of the new kaon spectrometer (HKS) and 2) a new electron spectrometer's configuration is adopted (tilt method). 
Employing the proposed new experimental configuration and with the HKS spectrometer, we expect to achieve hypernuclear yields more than one order of magnitude higher and the signal-to-accidental ratio one order of magnitude better simultaneously with the twice better energy resolution compared to the previous E89-009 experiment. The proposal of the new experiment with the HKS and the tilt method was accepted by JLab PAC19[3].

\subsection{A new high resolution kaon spectrometer (HKS)}

The HKS consists of two quadrupole magnets Q1 (8.5 tons) and Q2 (10.5 tons) and one dipole magnet D (210 tons). Due to two degrees of freedom of the quadrupole doublet, the horizontal and vertical focusing can be simultaneously adjusted. The HKS is designed to achieve both $2 \times 10^{-4}$ momentum resolution and $16 \mathrm{msr}$ solid angle acceptance at the same time when it is used with the Splitter. It means that the HKS spectrometer will have 3 times greater solid angle and twice better resolution than the previous kaon spectrometer simultaneously.

The HKS is positioned at an angle of $7^{\circ}$, covering from 0 to 14 degrees, with respect to $1.2 \mathrm{GeV} / c$ zero degrees scattered particle to avoid high-rate positively charged particles at 0 degrees, mostly positrons. Basic parameters for HKS are summarized in table 1.

Table 1

Specification of the HKS

\begin{tabular}{ll}
\hline Configuration & Q-Q-D and horizontal $70^{\circ}$ bend \\
Central momentum & $1.2 \mathrm{GeV} / c$ \\
Dispersion & $4.7 \mathrm{~cm} / \%$ \\
Momentum acceptance & $\pm 12.5 \%(1.05-1.35 \mathrm{GeV} / c)$ \\
Momentum resolution $(\Delta p / p)$ & $2 \times 10^{-4}$ \\
Solid angle & $30 \mathrm{msr}$ without Splitter \\
& $16 \mathrm{msr}$ with a splitter \\
Kaon detection angle & Horizontal : 7 degrees $\left(0-14^{\circ}\right)$ \\
Flight Path Length & $10 \mathrm{~m}$ \\
Maximum Magnetic Field & $1.6 \mathrm{~T}$ (normal conducting magnet) \\
\hline
\end{tabular}

The construction and the precise field mapping of the HKS magnets were carried out at Mitsubishi, Kobe (Japan) and they were disassembled for shipping. The HKS magnets arrived at JLab in November, 2003 and the pre-assembly at the test lab is in progress (Fig. 2).

\subsection{Tilt method and the electron spectrometer (ENGE)}

In the pilot (e,e'K) hypernuclear experiment, E89-009, the electrons associated with bremsstrahlung dominated the background in the scattered electron spectrometer (Enge split-pole spectrometer). These ultra-high-rate bremsstrahlung electron background limits the beam intensity and thus the hypernuclear yield. Therefore, it is essential how we can 


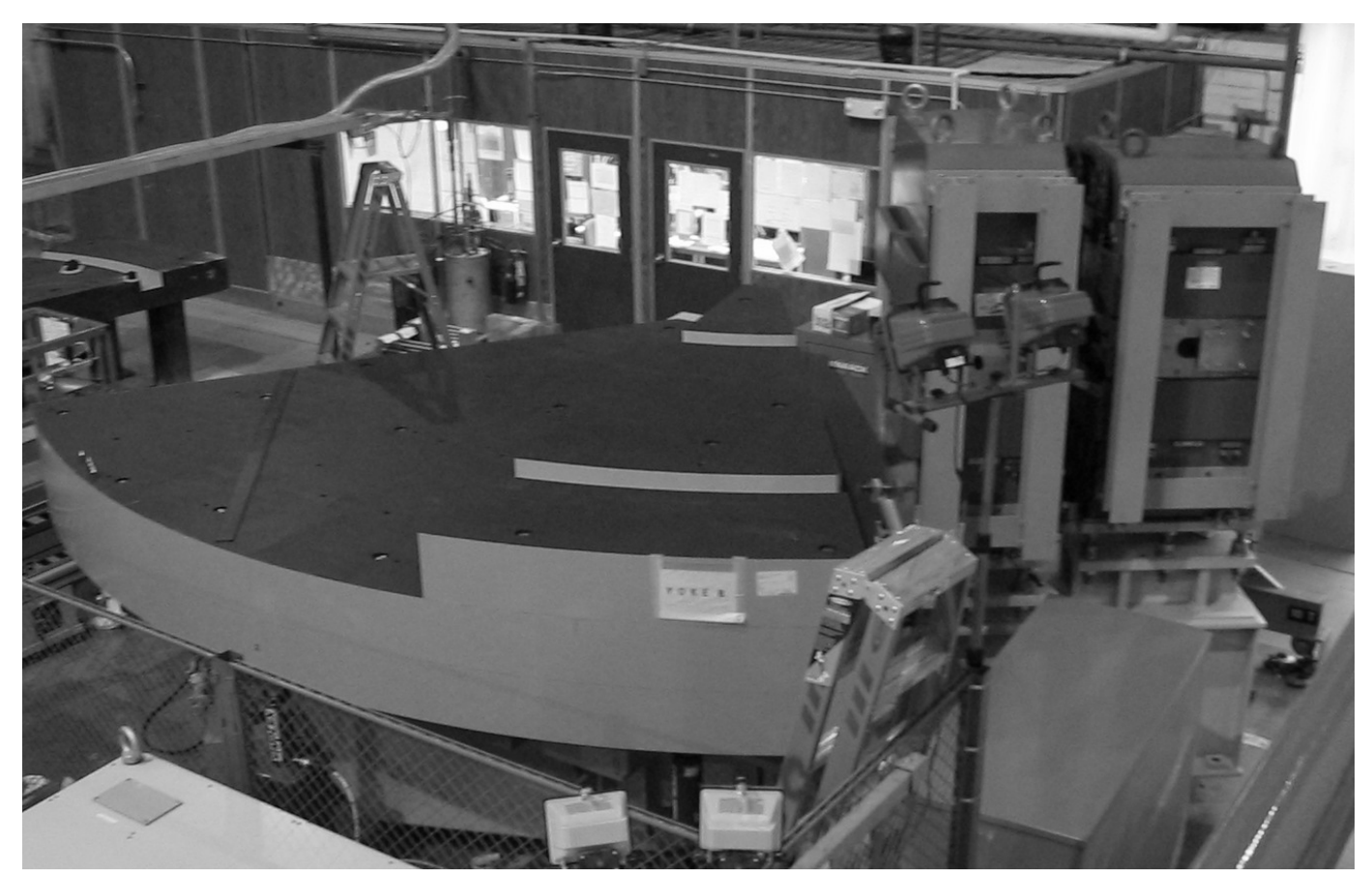

Figure 2. HKS pre-assembly at JLab test lab (Dec, 2003). Lower part of the HKS-D magnet and installation of Q1 and Q2 were finished. From right to left, Q1, Q2 and the lower part of D can be seen. After the fiducialization, the upper part of the magnet will be assembled.

suppress the bremsstrahlung background.

We took a particular note of the difference of the angular distributions between bremsstrahlung electrons and scattered electrons associated with the virtual photons which contributes to the kaon production. Both of electrons distribute very forward, however the bremsstrahlung electrons do more than those associated with the virtual photons. Therefore, we can avoid the extremely high rate electrons originating from bremsstrahlung and $M \phi l l e r$ scattering by the tilt of the ENGE spectrometer off the bending plane of the Splitter magnet (tilt method). However, increasing the tilt angle, the number of the accepted virtual photons will decrease. We need to take following processes into account to optimize the tilt angle:

1. virtual photons associated with hypernuclear production,

2. electrons associated with bremsstrahlung, and

3. $\mathrm{M} \phi$ ller scattering electrons.

Figure 3 shows the electron scattering angle distributions at the target. The electron distributions associated with bremsstrahlung, virtual photons and $\mathrm{M} \phi$ ller scattering are plotted. Since the beam energy is fixed, the scattering angle and momentum for M $\phi$ ller scattering electrons have one to one correspondence, and thus a ring shaped distribution results from the $M \phi$ ller scattering within momentum acceptance of the ENGE spectrometer. 
Using the RAYTRACE optics code, the events which passed through the tilted ENGE spectrometer without hitting the pole or collimators were selected. Figure 3 shows that the acceptance of the tilted ENGE spectrometer locates just outside of the M $\phi$ ller ring, and thus hyper-forward bremsstrahlung background and $\mathrm{M} \phi$ ller electrons are blocked while the electrons associated with virtual photons pass through the ENGE spectrometer.

Figure 4 shows the tilt angle dependence of the rates for those processes. Defining the figure of merit as Signal (virtual photon flux)/ square root of the background (M $\phi$ ller + bremsstrahlung), the ENGE tilt angle at about 8 degrees is optimum. After detailed optimization of the tilt angle and vertical offset of the ENGE spectrometer from the splitter dispersion plane, we set the ENGE tilt angle at 7.75 degrees which selects the electron scattering angle of $60 \sim 100 \mathrm{mr}\left(3.4 \sim 4.6^{\circ}\right)$.

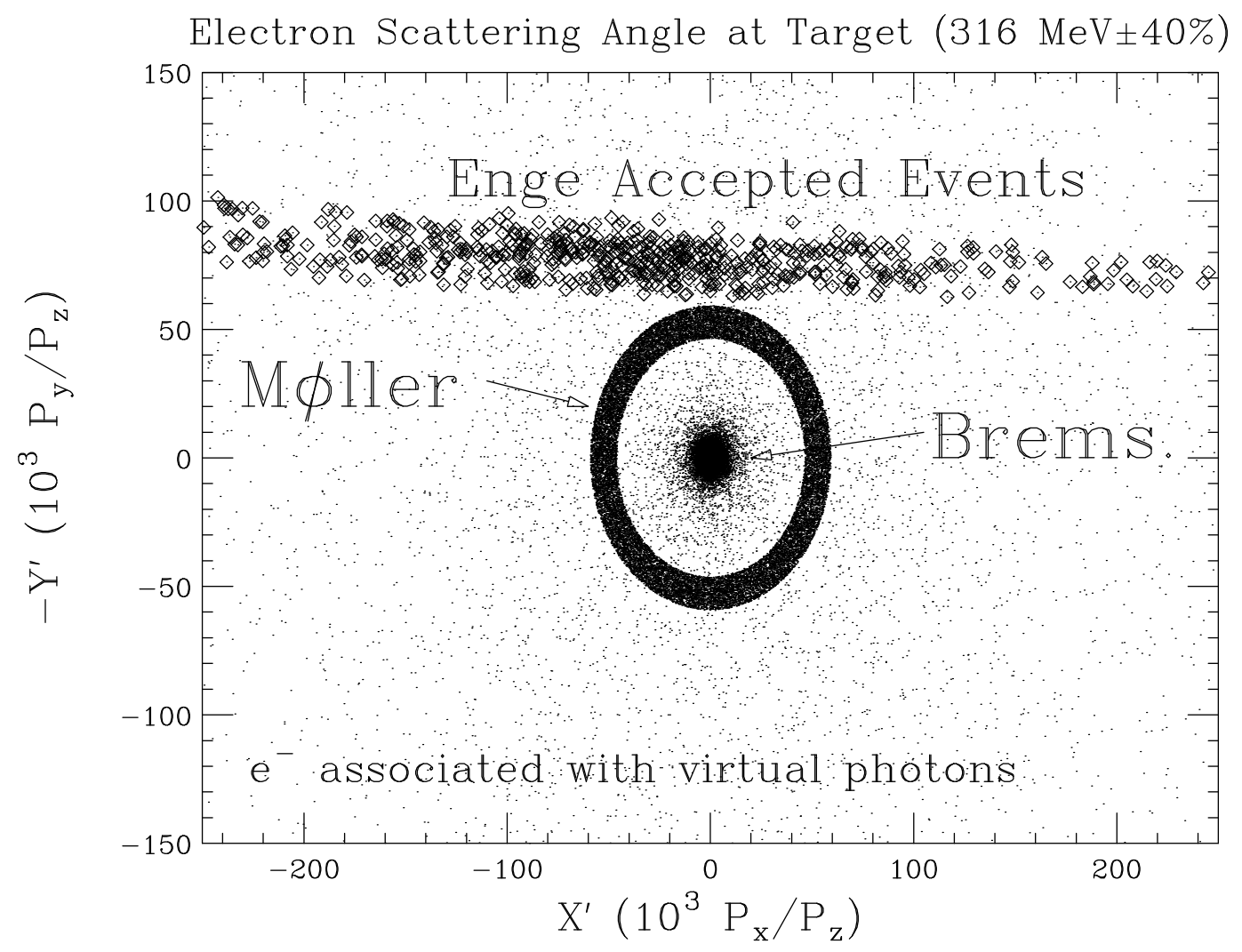

Figure 3. The scattering angle of the electrons $(p=316 \pm 40 \% \mathrm{MeV} / c)$ at the target calculated by the program RAYTRACE. The very forward bremsstrahlung electrons and $\mathrm{M} \phi$ ller electron rings are observed. The acceptance of the $7.75^{\circ}$ tilted ENGE spectrometer locates just outside of the M $\phi$ ller ring.

The tilt method reduces drastically $\left(\sim 10^{-4}\right)$ the electron rate at the electron detectors. In the E89-009 experiment, the electron rate was over $200 \mathrm{MHz}$ and it limited the beam intensity and the target thickness. The virtual photon yield is also reduced by the tilt 


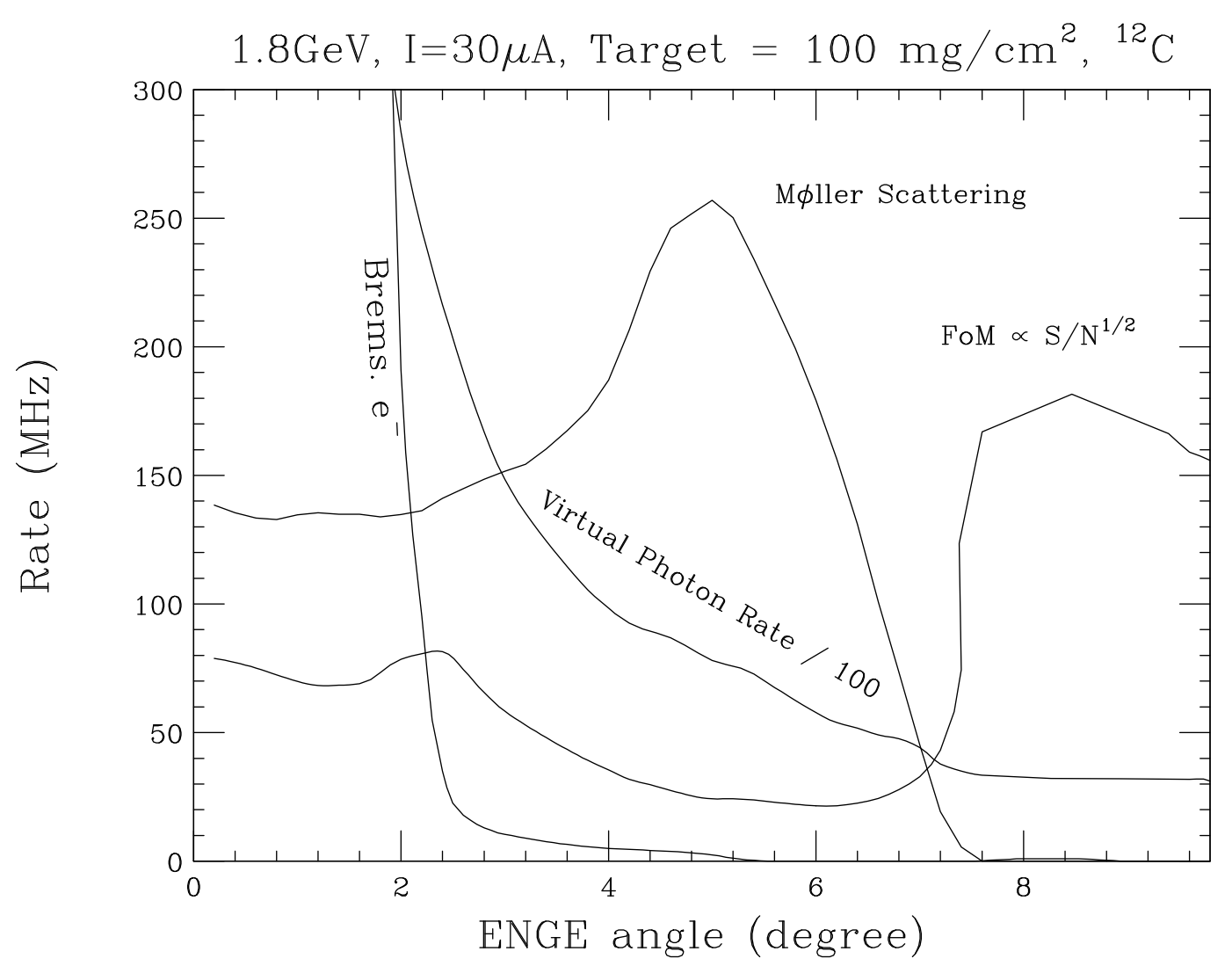

Figure 4. ENGE tilt angle dependence of the expected rates. The beam current of $30 \mu \mathrm{A}$ and carbon target of $100 \mathrm{mg} / \mathrm{cm}^{2}$ are assumed. Figure of merit (FoM) is defined as $S / N^{1 / 2}$, where $S$ is the virtual photon flux and $N$ the sum of bremsstrahlung electrons and M $\phi$ ller scattering electrons. For readers' eyes, the virtual photon flux is multiplied by 0.01 and FoM by an arbitrary factor.

method, however, it can be easily recovered by increasing the beam intensity and target thickness. The tilt method will reduce the electron rate from $200 \mathrm{MHz}$ to a few $\mathrm{MHz}$ for ${ }^{12} \mathrm{C}$ target even with 5 times thicker target and $\sim 50$ times more intense beam. It should be noted that the tilt method opens a chance to measure the hypernuclear spectra for the higher $Z$ targets through the $\left(\mathrm{e}, \mathrm{e}^{\prime} \mathrm{K}^{+}\right)$reaction. The reduction of the electron background is essential for the heavier target, since the bremsstrahlung rate increases roughly in proportion to $Z^{2}$.

\section{Development of the detector packages}

\section{Kaon spectrometer}

The HKS detector package is designed to identify $\mathrm{K}^{+} \mathrm{s}$ from $\mathrm{p}, \pi^{+}$, $\mathrm{e}^{+}$backgrounds with a total rate of a few $\mathrm{MHz}$ at maximum and to measure their momentum with an accuracy of $2 \times 10^{-4}(\mathrm{FWHM})$. In order to realize the above requirements, the HKS 
detector package consists of one pair of drift chambers (HDC), three planes of time-offlight counters (HTOF), three planes of aerogel Čerenkov counters (AČ), and two planes of water Čerenkov counters (WČ). A schematic view of the HKS detector package is shown in figure 5.

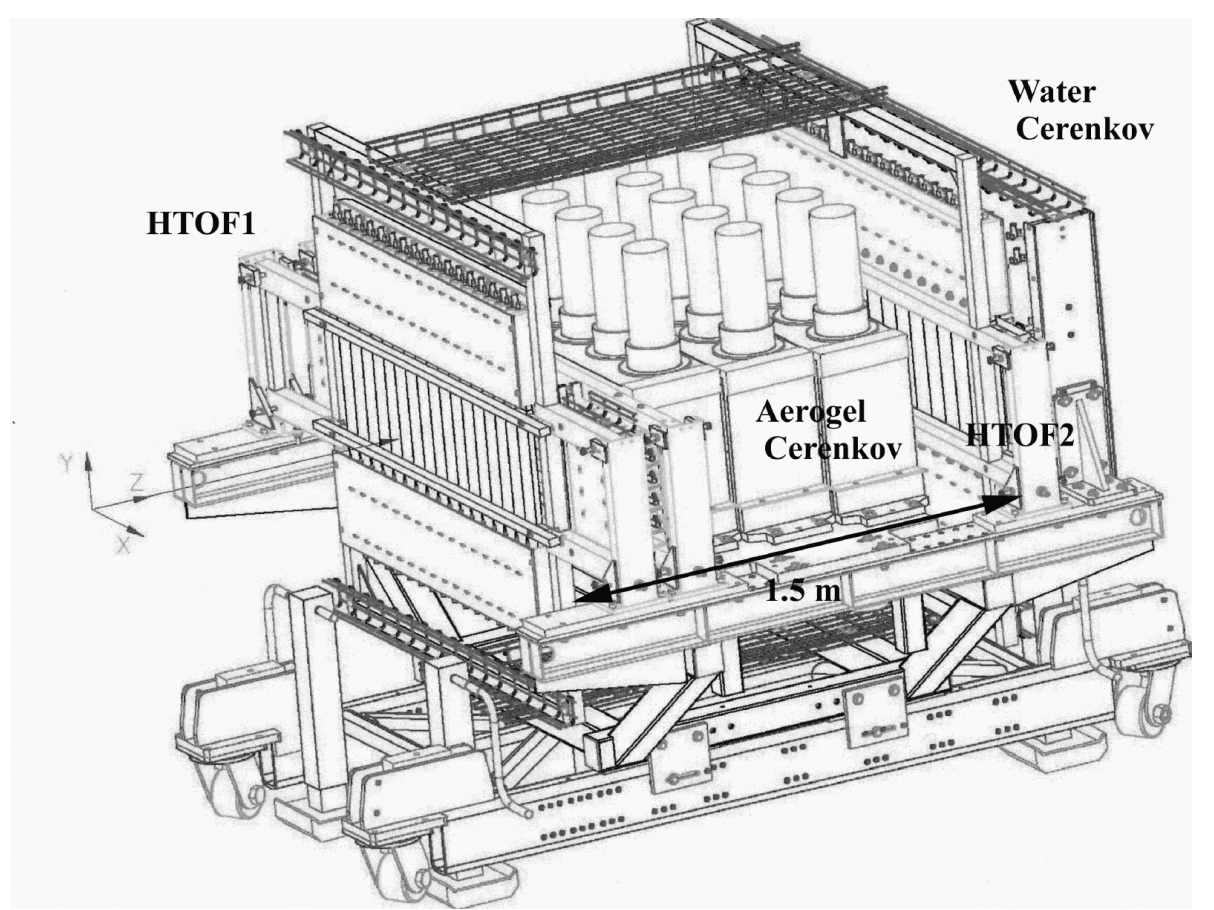

Figure 5. Schematic view of the HKS detector package. Two drift chambers (HDC) are placed with a space of $1 \mathrm{~m}$ just after the HKS-D magnet (far left, not drawn here). Downstream of the HDCs, a movable table, which is shown in this figure, is located. On the table, a start counter array for the time of flight measurement (HTOF1), aerogel Čerenkov counters (AČ), a stop counter array (HTOF2) and water Cerenkov counters are placed. The flight path for TOF measurement is $1.5 \mathrm{~m}$.

The HDC is basically the same type of the drift chamber used for SOS (Short Orbit Spectrometer) at Hall C. HDC1 and HDC2 are placed with $1 \mathrm{~m}$ space and each of them consists of 6 layers $\left(x x^{\prime} u u^{\prime} v v^{\prime} ; u\right.$ and $v$ planes are respectively tilted by 30 and $\left.-30^{\circ}\right)$. The HTOF counters are designed to achieve a time resolution of 70 ps (rms) for $1.35 \mathrm{GeV} / c$ $\mathrm{K} / \pi$ separation with a flight length of $1.5 \mathrm{~m}$. Matsushita Electric's hydrophobic aerogel $(n=1.050)$ is used for the AČ to discriminate $\pi \mathrm{s}$ from $\mathrm{Ks}$ in the trigger level. In order to separate Ks and protons, water Čerenkov counters (WČ, $n=1.33$ ) with a wave length shifter (animo-G-salt) are used.

In order to suppress the overkill of the precious $\mathrm{K}^{+}$and to achieve high rejection power for $\pi^{+}$and protons in the trigger level, the counters are segmented (TOF1 into 17, TOF2 into 18, AC into 7 and WC into 12). The first level trigger is made from the grouping of those segmented counters, as $\mathrm{K}$ trigger $=\sum_{i} \mathrm{TOF} 1_{i} \cdot \overline{\mathrm{AC}}_{i} \cdot \mathrm{WC} \check{C}_{i} \cdot \mathrm{TOF} 2_{i}$ (subscript of 
$i$ means the $i$-th group of those segmented counters). The grouping is determined by a Monte Carlo simulation before the beamtime, but there might be a possibility to change the grouping or trigger scheme during the beamtime.

In order to minimize the effort to change the hard-wired triggers, we developed a special trigger module (Tohoku Universal Logic, TUL-8040) which uses a Field Programmable Gate Array chip (Altera APEX20K300EQC240-1X). The module can be programmed with the VHDL language and the programmed logic can be checked with a circuit simulator. The grouping condition can be easily changed during the beamtime by up-loading the pre-tested programs.

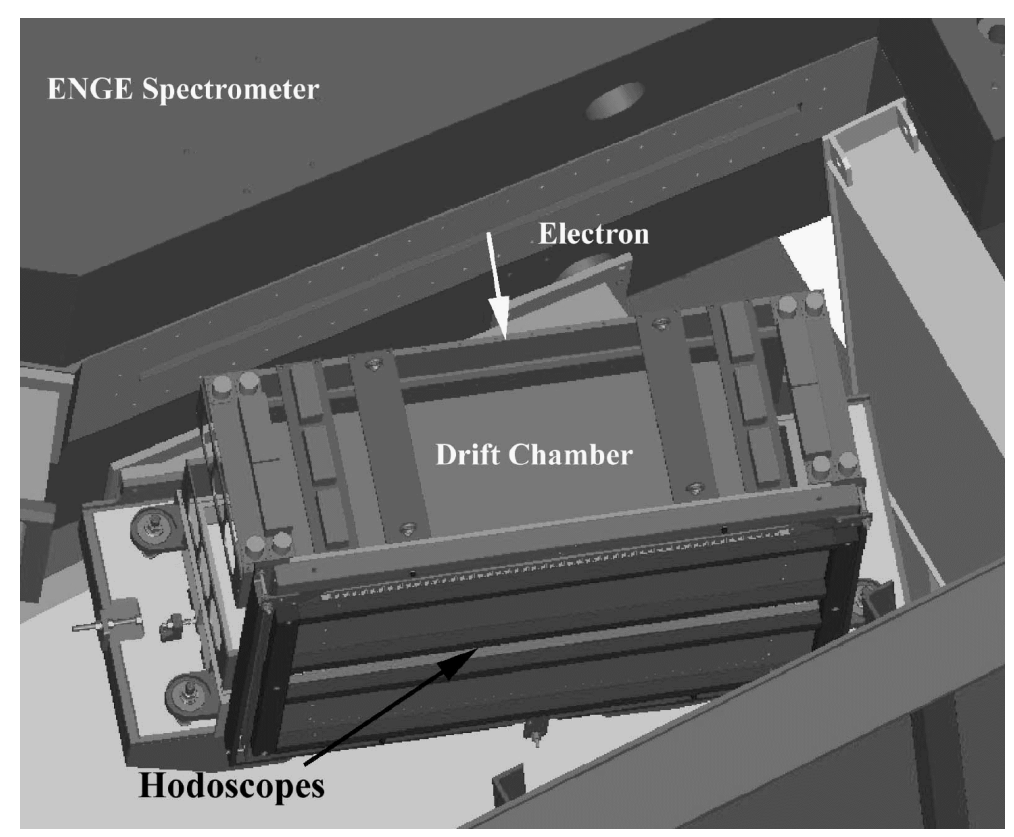

Figure 6. Schematic view of the ENGE detector package. The scattered electrons are bent by the ENGE magnet and enter to the honeycomb-cell structured drift chamber (EDC). The vacuum extension is not drawn for a clear view. The EDC covers the effective area of $100 \mathrm{~cm} \times 12 \mathrm{~cm}$. Just downstream of the EDC, two layers of scintillator hodoscopes are located. The ENGE detector package is shielded by lead wall to avoid background from the target.

\section{Electron spectrometer}

In the E89-009 experiment, the ENGE spectrometer is placed in the Splitter's dispersion plane, and thus the position measurement gives enough momentum resolution without angular information. However, the tilt method distorts its original optical nature and it is essential to measure not only the particles hit position but also the angle of them at the focal plane of the spectrometer. We developed a new honeycomb-cell structured drift 
chamber (EDC) in order to measure the position and angles of the scattered electrons with angles distributed from -5 to $40^{\circ}$ at the exit of the ENGE spectrometer. The EDC has ten layers $\left(x x^{\prime} u u^{\prime} x x^{\prime} v v^{\prime} x x^{\prime} ; u\right.$ and $v$ planes are respectively tilted by 30 and $-30^{\circ}$.) and all layers are installed in one chamber without windows to minimize the multiple scattering effect. The chamber is designed to achieve position resolution of $200 \mu \mathrm{m}$ per plane and angular resolution of $1.5 \mathrm{mrad}$ for $300 \mathrm{MeV} / c$ electrons.

The tilt method reduces the electron rate down to a few $\mathrm{MHz}$, but it is still high rate. Therefore, a plastic scintillation counter to measure the electron timing should be highly segmented. Just behind of the EDC, two layers of the plastic scintillation counter hodoscope (25 segmentation / layer) are placed for the timing measurement. Schematic view of the ENGE detector package is given as figure 6 .

\section{Detector beam test}

Prototypes of aerogel and water Čerenkov counters, mass production version of the HKS TOF counters, ENGE hodoscopes are tested with $1.05 \sim 1.35 \mathrm{GeV} / c$ unseparated p, $\mathrm{K}^{+}, \pi^{+}, \mathrm{e}^{+}$beam at KEK-PS (T494, T500, T530), Tsukuba, Japan (Fig. 7). The EDC (honeycomb-cell drift chamber) is also beam tested at LNS, Tohoku University with $\mathrm{e}^{+} \mathrm{e}^{-}$ pairs converted from the tagged photons.

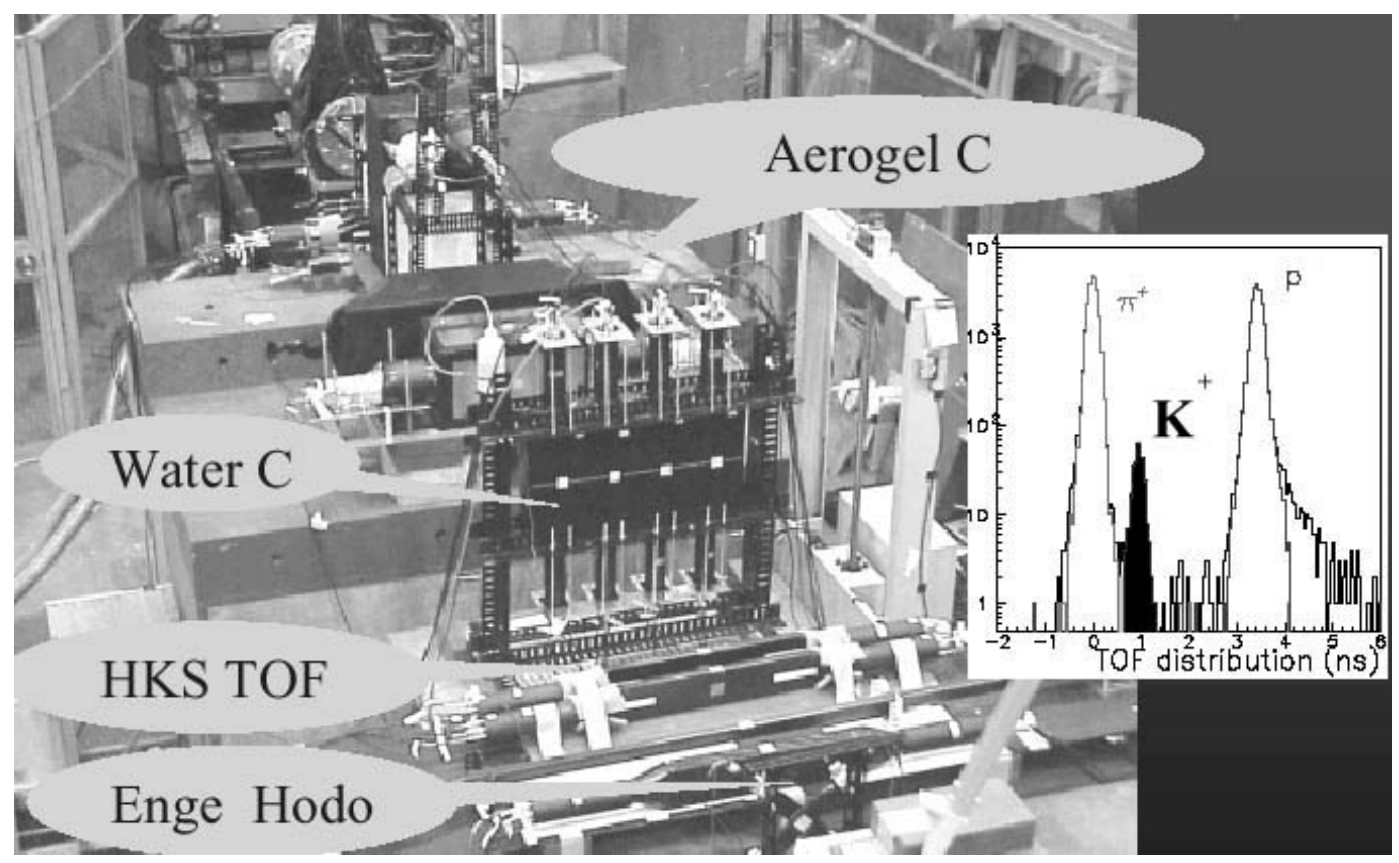

Figure 7. Counter beam-test setup at the T1 beamline of the KEK $12 \mathrm{GeV}$ proton synchrotron. $\mathrm{K}^{+} \mathrm{s}, \pi^{+} \mathrm{s}$, protons were separated with TOF information and $\mathrm{e}^{+} \mathrm{s}$ are identified with $\mathrm{CO}_{2}$ gas Čerenkov counter. With those particle ID information, aerogel and water Cerenkov counters' responses were investigated.

The fabricated counters and EDC were checked to achieve the required performance 
with beams, and they have been transported to the JLab EEL building for the final tune.

\section{Summary}

A new improved hypernuclear spectroscopy up to medium heavy target with a newly developed High resolution Kaon Spectrometer (HKS) and a new configuration of the electron spectrometer (tilt method) is planned at the JLab Hall-C. The energy resolution will be improved by a factor of two; the hypernuclear yield and the signal to noise ratio will be improved by a factor of 50 and 10 , respectively.

The HKS magnets fabrication and field map were finished in Japan and they were safely transported to JLab. Pre-assembly is now in progress at the test lab, JLab. Detector development and beam test were finished. They are now under the final tune at the EEL building of JLab. The preparation for the experiment is in progress as planned. We will be ready for the beam in year 2004 .

\section{REFERENCES}

1. T. Miyoshi, et al., Phys. Rev. Lett., 90, 232502 (2003).

2. SAPHIR collaboration, Phys. Lett., B 445, 20 (1998).

3. O. Hashimoto, L. Tang, J. Reinhold, S.N. Nakamura, et al., E01-011 Proposal submitted to JLab PAC19 (2001); S.N. Nakamura et al., Physics with GeV Electrons and Gamma-rays (Eds. T.Tamae et al.), Universal Academy Press, (2001) 113. 